\title{
A study of salmonella carriage among asymptomatic food-handlers in southern Ethiopia
}

\author{
Misganaw Birhaneselassie ${ }^{1, ~ *}$, David Williams ${ }^{2}$ \\ ${ }^{1}$ Hawassa University, College of Medicine and Health Sciences, PO box 1560 Hawassa, Ethiopia \\ ${ }^{2}$ Department on Microbiology and Infection Control, Nevill Hall Hospital, Abergavenny, Monmoutnshire NP7 7EG
}

Email address:

misganawb@yahoo.com (M. Birhaneselassie), d.awilliam08@btinternet.com (D. Williams)

To cite this article:

Misganaw Birhaneselassie, David Williams. A Study of Salmonella Carriage among Asymptomatic Food-Handlers in Southern Ethiopia. International Journal of Nutrition and Food Sciences. Vol. 2, No. 5, 2013, pp. 243-245. doi: 10.11648/j.ijnfs.20130205.15

\begin{abstract}
Background: Salmonella is the most frequently reported cause of food borne illness in Ethiopia. Objective: The aim of this study was to screen food handlers in the Dilla area of Southern Ethiopia and to assess the scale of carriage of Salmonella species and to offer prophylaxis followed by re-testing and advice on personal and food hygiene as appropriate. Method: Stool samples were collected from a total of 107 food handlers from various establishments in the area. Enrichment culture using Selenite broth and sub-culture on to Deoxycholate Citrate agar was used followed by identification to genus level by use of a commercially available biochemical kit and polyvalent antisera. Result: Salmonella was isolated from one food handler only and this person could not be traced having left her employment and not having left a forwarding address. Conclusion: As food poisoning is a main issue in Ethiopia further epidemiological research, to monitor those found to be infected is mandatory.
\end{abstract}

Keywords: Salmonella, Screening, Food handlers, Asymptomatic

\section{Introduction}

Salmonella is the most frequently reported cause of food borne illness, worldwide. WHO estimated that at least 600,000 deaths occurred each year due to typhoid fever. Salmonella infection most commonly occurs in countries with poor standards of hygiene in food preparation and handling and where sanitary disposal of sewage is lacking. It mainly occurs in the tropics and sub tropics in Africa, India, Pakistan South East Asia and South America [1-7].

Food borne salmonellosis often follows consumption of contaminated animal products such as raw meat, poultry and eggs. Not washing fresh fruits and vegetables before eating them, as well as not thoroughly cleaning work surfaces used to prepare raw meat and other foods in the kitchen can also be source of Salmonella. Food can also be contaminated by food handlers who do not thoroughly wash their hands with soap after handling raw meat or after using the bathroom [4].

Studies indicated the widespread occurrence and distribution of Salmonella in Ethiopia. In recent years the number of out breaks of Salmonella in humans has increased considerably in the country. Much more is known now about the extent of food borne illness and how severe it can be, not just in terms of acute illness, but also in terms of long term consequences. Studies indicated various percentages of Salmonella isolates in towns of Ethiopia. Moreover, high percentages of $S$. typhi isolates have been found to be resistant for antimicrobial agents $[8,9,10]$. In addition, the very young, elderly and immunocompromized individuals are particularly more susceptible to Salmonella infections at a lower infective dose than healthy adults. This is more important in developing countries such as Ethiopia where HIV/AIDS is highly prevalent and Salmonella is an important opportunistic infection in HIV/AIDS patients [11].

In Ethiopia, minced beef is usually used for the preparation of a popular traditional Ethiopian dish known as locally "KITFO" and most of the time it is consumed raw or medium cooked. The habit of raw meat consumption and the presence of Salmonella in minced beef indicate, in addition to the poor hygienic standards in food handling in the country, the presence of great public health hazards of Salmonella [6].

Moreover, about $2-5 \%$ of those who contract typhoid fever become chronic carriers, as bacteria persist in the biliary tract after symptoms have resolved [7]. Therefore, carrier states of humans are of concern to the food manufacturing and food service institutions because of the 
perceived risk of contamination of food by infected food handlers and the risk of food borne disease outbreaks [6].

Strategies for reducing food borne illness require a comprehensive farm-to-table approach, while Salmonella contamination from food handlers has been shown to make a significant contribution to human food borne illness in several developing countries [11].

The goal of this project is to carry out a preliminary survey of Salmonella from healthy food handlers. The project also helps as a health education tool to elucidate the main pathways by which consumers are exposed to Salmonella and helps the Ministry of Health of Ethiopia to achieve its strategic goal of reducing the incidence of food borne illness in the country.

\section{Materials and Methods}

This survey was undertaken in 2002 with material and professional support from UK health link partners to Southern Ethiopia (www.ethiopiagwentlink.org). This was part of a practical training exercise for laboratory science and sanitarian students at Dilla College.

Food handlers who had a direct contact with food and food delivery to people in large establishments were selected as study participants. It was also arranged that health education and sanitary advise to be given by students and instructors from the College.

A number of food handles were approached by students of Medical Laboratory Technology under supervision of instructors and lecturers from the Dilla campus of the formerly known Debub University. The source of these were namely Dilla College campus 47, Dilla prison 11, Dilla hospital 3, Hotels and Butchery 46, a total of 107 food handlers were screened in the survey. These sources of food handlers from large establishments were a concern because a food handler positive for Salmonella could pass it for many people at a time.

Identifiers used were the name, date of birth or age (where known) and the name of the establishment. Overall, adults from age group of 20-40 were involved in the study. Majority of study participants were illiterate and were females.

Stool samples were collected from 107 of these using sterile containers over a two week period. A small piece of each stool sample was inoculated in to Selenite broth and incubated for 16 to 24 hours at $37^{\circ} \mathrm{C}$. Each Selenite broth was sub cultured on to Deoxycholate Citrate Agar (DCA) and incubated $37^{\circ} \mathrm{C}$ for a further 16 to 24 hours. Non-lactose fermenting colonies were tested using oxidase reagent and urea agar slopes. Oxidase and urease negative colonies were further identified using Biomerieux API 10S kits. Isolates identified as presumptive Salmonella species were tested with polyvalent $\mathrm{O}$ (somatic) and $\mathrm{H}$ (flagellar) antisera. Any food handler identified as a Salmonella carrier was to be offered a course of Ciprofloxacin and re-tested after finishing the course of antibiotics.

Ethical clearance was obtained from Dilla Health Science
College. Informed consent was obtained from every food handler. Support letter was sent to each facility from Dilla College for cooperation in sample collection and health education.

\section{Results}

Of the 107 food-handlers tested 1 was identified as being a Salmonella earner $(0.93 \%)$. The person (female) identified positive was working in a hotel. Unfortunately there was no microbiological analysis performed in the food from the hotel, colleagues from the hotel were negative for the test. On a further visit to this person's workplace to offer advice and a course of antibiotics, instructors from the University were told that she had left her employment and had not left a forwarding address and therefore could not be traced. It is not known whether this person obtained further employment as a food-handler.

\section{Discussion}

It is believed that this study is the first of its kind to be carried out in Southern Ethiopia. Definitive identification of more Salmonella isolates was not possible given the facilities available but it is believed that the methodology used was sufficient for identification to genus level. The percentage positive obtained compares favorably with similar studies in other developing countries. In Lima, Peru [2] 29 out of 1,931 (1.5\%) food handlers proved positive for Salmonella carriage. Of these $4(0.2 \%)$ were found to be $S$. typhi carriers. In Irbid, Jordan [3], out of 283 food-handlers screened, 17 (6\%) were Salmonella carriers. In Ghana [12], 258 asymptomatic food vendors were screened and Salmonella was isolated from 6 (2.3\%). In India [5], among 35 individuals screened $6(17.14 \%)$ were positive for Salmonella.

Similar study indicated comparable percentage of positivity for Salmonella in Ethiopia. In Bahir Dar, 1.6\% food handlers out of 384 were found positive for S. typhi [8]. However, in Gondar town, Andargie reported that no Salmonella species were isolated in food handlers [9]. Likewise, study undertaken in Hawassa revealed 8.1\% (22) S. typhi positive food handlers out of 272 from blood sample by Widal test, while there was no Salmonella identified by microbiological methods from stool specimen in this study [13]. The methodology applied in this survey was comprehensive in that it used serial of culture, biochemical and serological methods to confirm the Salmonella colonies.

\section{Conclusion}

Routine testing of food handlers in Southern Ethiopia would be difficult and if the carriage rate detected is typical, probably not necessary. Further studies of Salmonella carriage in Southern Ethiopia should be possible given the development taking place within the university and the Referral Hospital at Hawassa. The relatively small numbers 
of food handlers tested compared with the majority of the other studies referred to indicate that a larger number of individuals should be tested to gain an accurate picture of the true carriage rate.

\section{Acknowledgements}

The study was the result of collaboration between partners in the Gwent Healthcare/Southern Ethiopia link which was set up under the auspices of the Tropical Health Education Trust (THET). The project was supported by the link for supply of laboratory materials and also by donations of laboratory materials from Oxoid UK and Biomerieux Ltd.

It was used as a teaching tool to help Medical Laboratory Technology students at the Dilla campus of the university to gain practical experience of microbiological techniques. The students were responsible for carrying out much of the work including specimen collection and were supervised throughout by instructors from the university as well as link partners from the UK.

The authors wish to acknowledge the contributions of Dr Aberra Alemneh and Mr Sunirmal Ghosh for organizational help, Mr David Sanders for assistance in preparing training material and Mr Moges Derbe for technical assistance.

\section{Competing Interests}

The authors declared that they have no competing interest to disclose in this research.

\section{References}

[1] World Health Organization Manual for the laboratory identification and antimicrobial susceptibility testing of bacterial pathogens of public health importance in the developing world. Geneva: WHO; 2003: 382-381.

[2] Lanata CF, Tafur C, Benavente L, Gotuzzo E, Camllo C. Detection of Salmonella typhi carriers in food handlers by vi serology in Lim, a Peru. Bull Pan Am Health Organ 1990;
24(2):177-82.

[3] al-Lahham AB, Abdu- Saud M,Shehabi AA. Prevalence of Salmonella, Shigella and Intestinal parasites in food handlers in Irbid, Jordan. J Diarrhoeal Dis Res 1990; 8(4); 160-2.

[4] World health organization. Health surveillance and management procedures of food-handling personnel. Geneva: WHO; 1989. p. 7-36.

[5] Senthikumar B, Prabakaran G. Multi drug Resistant salmonella typhi in Asymptomatic Typhoid carriers among food handlers in Namakkal District, Tamil Nadu. Indian J Med Microbiology 2005; 23:92-94.

[6] Muleta D, Ashenafi M. Salmonella, Shigella and growth potential of other food- borne pathogens in Ethiopia street vended foods. East African Medical Journal 78 (I I) November 2001

[7] World Health Organization. International Travel and Health: WHO, 2010

[8] Andargie G, Kassu A, Moges F, Tiruneh M, Henry K. Prevalence of Bacteria and Intestinal Parasites among Food-handlers in Gondar town, North West Ethiopia. J Health Popul Nutr 2008; 26(4):451-455.

[9] Abera B, Biadegelgen F, Bezabih B. Prevalence of Salmonella typhi and intestinal parasites among food handlers in Bahir Dar Town, Northwest Ethiopia. Ethiop. J. Health Dev 2010; 24(1)

[10] Yismaw G, Negeri C, Kassu A, Tiruneh M and Mulu A. Antimicrobial resistance pattern of Salmonella isolates from Gondar University Hosipital, North West Ethiopia. Ethiop Pharm J 2007; 25(2):85-90.

[11] Catherine E, Woteki, Sandra L, Facinoli, Daniel S. Keep Food Safe to Eat Healthful food must be safe as well as nutritious. J. Nutr 2001; 1319 (2) 502-509.

[12] PK Feglo, EH Frimpong, M Essel- Ahun. Salmonellae carrier status of food vendor in kumasi, Ghana. East African Medical Journal 2004; 81(7):358-361.

[13] Desta M. "Prevalence of Salmonella and Shigella among Food Handlers in Catering Establishments in Hawassa University, Hawassa, Ethiopia, Addis Ababa University 2010" unpublished. 